\title{
Basal-bolus Therapy in Patients with Type 2 Diabetes Mellitus in the UK: Patient Characteristics, Treatment Patterns and the Effect of Switching to Premixed Insulin
}

\author{
Kate van Brunt - Bradley Curtis - Tibor Ivanyi - Elemer Balogh • \\ Corina Chalkiadaki - Sharon MacLachlan · David Neasham • \\ Mireia Raluy-Callado
}

Received: July 13, 2016 / Published online: October 31, 2016

(c) The Author(s) 2016. This article is published with open access at Springerlink.com

\section{ABSTRACT}

Introduction: Increasing emphasis is being placed on insulin use among patients with type 2 diabetes mellitus (T2DM). Basal-bolus (BB) therapy is regarded as the gold standard, but a high frequency of injections and the general complexity of this therapy are seen as barriers in real-world practice. Here we describe the characteristics and treatment patterns of patients with $\mathrm{T} 2 \mathrm{DM}$ receiving $\mathrm{BB}$ in the $\mathrm{UK}$, with specific focus on those switching to a simplified regimen of premixed insulin.

Methods: Patients with T2DM receiving BB from 1 January 2005 were identified from the Clinical Practice Research Datalink.

Enhanced content To view enhanced content for this article go to http://www.medengine.com/Redeem/ 3317F0600150A08D.

Electronic supplementary material The online version of this article (doi:10.1007/s13300-016-0209-4) contains supplementary material, which is available to authorized users.

K. van Brunt · B. Curtis · T. Ivanyi · E. Balogh

Eli Lilly and Company, Wood Manor, Surrey, UK

C. Chalkiadaki $\cdot$ S. MacLachlan · D. Neasham .

M. Raluy-Callado $(\bowtie)$

Evidera, Hammersmith, UK

e-mail: Mireia.Raluy@evidera.com
Characteristics were described at treatment initiation or on 1 January 2005, and treatment patterns were assessed at 12 months of follow-up. Clinical factors were compared in two groups of patients who while receiving BB had one haemoglobin A1c (HbA1c) measurement of $\geq 53 \mathrm{mmol} / \mathrm{mol} \quad(7.0 \%)$ and remained either on $\mathrm{BB}$ or switched to a premixed insulin regimen.

Results: Study criteria were met by 12,060 subjects (mean age 59 years; duration diabetes 12.4 years). The mean HbA1c concentration was $76 \mathrm{mmol} / \mathrm{mol}$ (9.1\% of patients), and $84.0 \%$ of patients were overweight. At 12 months of follow-up, $74.5 \%$ of the patients who had started BB remained on it. While on BB, 8835 patients had a HbA1c measurement of $\geq 53 \mathrm{mmol} / \mathrm{mol}$ ( $7.0 \%$ of all patients); of these, 95.9\% remained on $\mathrm{BB}$ and $4.1 \%$ switched to premixed insulin. Mean HbA1c levels were consistently higher for patients who switched to premixed insulin than for those who remained on BB, but the levels remained relatively unchanged over time.

Conclusion: A large proportion of patients receiving insulin did not achieve good glycaemic control in clinical practice. A small 
subset with higher comorbidities and HbA1c levels switched to a simplified regimen. Little evidence was found that type of insulin therapy was associated with meaningful changes in key clinical factors over time.

Funding: Eli Lilly and company.

Keywords: Basal-bolus therapy; Glycaemic control; Premixed insulin; Primary care

\section{INTRODUCTION}

The prevalence of diagnosed diabetes in the UK rose from 1.4 million in 1996 to almost 3.5 million in 2014. More than one in 16 people have diabetes, of whom $90 \%$ have type 2 diabetes mellitus (T2DM) [1, 2]. Increasing emphasis is being placed on the appropriate use of insulin among patients with T2DM, with the aim to control the blood glucose so that the haemoglobin A1c (HbA1c) level remains below the general target of $53 \mathrm{mmol} / \mathrm{mol}(7.0 \%)$ for adults on a drug associated with hypoglycaemia, with the optimal HbA1c targets determined by both patient and disease characteristics, as specified by diabetes management guidelines [3, 4]. Clarity on how to use insulin is important given evidence from studies in the UK showing that the timing of the initiation and intensification of insulin therapy is often suboptimal [5].

The UK National Institute for Health and Care Excellence (NICE) guidelines on T2DM [3] recommend initiating insulin therapy as the second intensification step. Depending on lifestyle and risk of hypoglycaemia, a number of choices are available to the patient. If the patient's HbA1c level is $\geq 75 \mathrm{mmol} / \mathrm{mol}(9.0 \%)$, then consideration should be given to starting both neutral protamine Hagedorn insulin and short-acting insulin administered either separately or as a premixed human insulin preparation. The guidelines suggest that adults using basal insulin be monitored to determine the need for treatment intensification with an additional short-acting (or premixed) insulin injection before meals (bolus insulin). Recent data provide evidence that therapy with both basal and prandial insulin results in better glycaemic control than basal insulin treatment alone $[6,7]$.

Few studies have examined the extent to which the NICE guidelines for insulin intensification are followed in primary care settings in the UK. Gallagher et al. [8] investigated the effect of financial incentives introduced in the UK in 2004 and found that proportionately more patients with T2DM received pharmacological treatment after the incentives were enacted; however, this study, did not examine clinical outcomes. A recent study by Khunti et al. [9] showed that amongst patients with an HbA1c level of $\geq 58 \mathrm{mmol} / \mathrm{mol}$ (7.5\%), 30.9\% had their regimen intensified, taking place on average 3.7 years after the initiation of insulin therapy. Barriers to initiation and intensification, such as the high injection frequency required with a basal-bolus (BB) insulin regimen and subsequent non-compliance with the treatment, [10, 11], have been identified [12]. A recent position statement of the American Diabetes Association (ADA)/European Association for the Study of Diabetes (EASD) shows that there are a number of possible options a patient with T2DM would have if non-insulin therapy is no longer effective, including basal insulin only, basal insulin + a rapid-acting (mealtime) insulin injection or premixed insulin therapy [4].

Premixed insulin, which requires fewer daily injections, may be an alternative [13]. A meta-analysis by Wang et al. [14] found that in insulin-naïve patients with T2DM, premixed 
insulin was equally effective as BB insulin in reducing HbA1c.

Publically available data in the UK documenting the treatment patterns and effectiveness of BB regimens in clinical practice are scarce. Therefore, we analysed data from a UK primary care database to address two main objectives. The first was to describe the demographic and clinical characteristics [age, comorbidities, HbA1c levels, body mass index (BMI), blood pressure] and treatment switching patterns of patients with T2DM who were treated with $\mathrm{BB}$ insulin. The second objective was to compare, among patients receiving a $\mathrm{BB}$ regimen who have not achieved glycaemic control, the changes in selected clinical factors between those who remained on their BB regimen and those who switched to premixed insulin.

\section{METHODS}

This study used longitudinal data from the Clinical Practice Research Datalink (CPRD) database, which integrates primary care records with prescription data and represents approximately $7.0 \%$ of the UK population. This is an appropriate source of data for this study as general practitioners (GPs) are the main healthcare providers for patients with T2DM in the UK $[15,16]$. The CPRD is a fully anonymised database using a random number as the patient identifier, and details on the CPRD have been previously published [17, 18]. The study objectives were addressed by analysing two specific, but related, samples that were drawn from patients diagnosed with T2DM any time before 1 January 2013. A diagnosis of T2DM was identified through disease-related Read codes and prescriptions of oral antidiabetic drugs (OAD); prescriptions for insulin were not included to avoid selecting patients with type 1 diabetes mellitus (see Electronic Supplementary Material).

The study protocol was reviewed and approved by the Independent Scientific Advisory Committee (ISAC) of the Medicines and Healthcare products Regulatory Agency database research (protocol number 13-220R).

\section{Treatment Patterns Sample}

The patient sample used to describe the demographic and clinical characteristics and analyse treatment patterns consisted of patients with T2DM who received at least one prescription of $\mathrm{BB}$ insulin concurrently between 1 January 2005 and 31 March 2012. The index date for this cohort was defined as the first $B B$ regimen identified in the study period or 1 January 2005, if the regimen was initiated prior to that date. The baseline period was defined as the 12-month period prior to the index date. Patients were followed for up to 12 months after the index date unless they died or transferred out of the GP practice first.

\section{Comparison Sample}

The sample of patients with T2DM who started a BB insulin regimen and had at least one HbA1c measurement of $\geq 53 \mathrm{mmol} / \mathrm{mol}$ $(\geq 7.0 \%)$ while receiving $\mathrm{BB}$ insulin was used to compare the characteristics and outcomes of those who remained on their BB regimen with those who switched to premixed insulin during the 12 months after their HbA1c $\geq 53 \mathrm{mmol} / \mathrm{mol}(\geq 7.0 \%)$ measurement. The index date for this sample was the first HbA1c measurement of $\geq 53 \mathrm{mmol} / \mathrm{mol}(\geq 7.0 \%)$ after the initiation of BB insulin therapy. 


\section{Study Measures}

Age, sex, smoking status and comorbidities previously analysed by Blak et al. [19] (cancer, cerebrovascular disease, congestive heart failure, coronary artery disease, depression, diabetic nephropathy, diabetic neuropathy, diabetic retinopathy, myocardial infarction and peripheral vascular disease) and the use of antidiabetic (biguanides, sulphonylureas, other oral drugs and glucagon-like peptide-1), antihypertensive, lipid-lowering and antithrombotic medications were measured during the baseline period according to relevant British National Formulary categories.

Clinical factors, HbA1c $(\mathrm{mmol} / \mathrm{mol}$ and percentage), BMI $\left(\mathrm{kg} / \mathrm{m}^{2}\right)$, blood pressure (systolic and diastolic, in $\mathrm{mmHg}$ ) and total cholesterol levels $(\mathrm{mmol} / \mathrm{L})$ were measured during the 12-month period prior to the index date, using the one measurement closest to the index date for the purposes of describing the sample. For the two groups in the comparison sample, these variables were assessed in five 90-day intervals beginning with 90 days prior to the index date and ending 365 days after the index date; the average of the available measurement per interval was used.

Patients could be classified as overweight (BMI 25 to $<30 \mathrm{~kg} / \mathrm{m}^{2}$ ) or obese (BMI $\geq 30 \mathrm{~kg} /$ $\mathrm{m}^{2}$ ), as having high blood pressure (systolic blood pressure $>140 \mathrm{mmHg}$ or diastolic blood pressure $>90 \mathrm{mmHg}$ ) and as having borderline-high $(5.2-6.2 \mathrm{mmol} / \mathrm{L})$ or high $(\geq 6.2 \mathrm{mmol} / \mathrm{L})$ total cholesterol.

Prescription data were used to classify patients in the treatment sample into one of five groups based on their insulin-use patterns: patients who started on BB and (1) remained on their BB regimen without any other insulin prescriptions, (2) switched to premixed (human or analogue type) insulin during the 90 days after stopping their BB regimen; (3) received premixed insulin while receiving their $\mathrm{BB}$ regimen; (4) switched from $\mathrm{BB}$ to another insulin regimen (not premixed); discontinued insulin treatment. Patients could use OADs while using insulin.

\section{Statistical Analyses}

Summary descriptive statistics [mean with standard deviation (SD), median with interquartile range (IQR), minimum and maximum] were computed for continuous variables, and number and percentage of patients in each category were computed for categorical variables. Student's $t$ test and one-way analysis of variance were used to compare continuous variables between two groups, or across more than two groups, and the Chi-square test was used to compare categorical variables across groups.

Analyses were conducted using SAS software (ver. 9.4; SAS Institute, Cary, NC).

\section{Compliance with Ethics Guidelines}

This study was approved by the ISAC of the Medicines and Healthcare products Regulatory Agency Database Research (protocol number 13_220). This article does not contain any new studies with human or animal subjects performed by any of the authors.

\section{RESULTS}

\section{Study Samples}

Overall, 210,778 people were identified in the CPRD database with T2DM diagnosed prior to 1 January 2013; of these, 12,060 people met our inclusion/exclusion criteria and received BB therapy on or after 1 January 2005 (36.5\% 
started BB therapy prior to 1 January 2005; $55.3 \%$ started BB therapy on or after 1 January 2005; the remainder had previously been on BB therapy, stopped and restarted after 1 January 2005). These 12,060 individuals formed the treatment pattern sample (Fig. 1). The sample

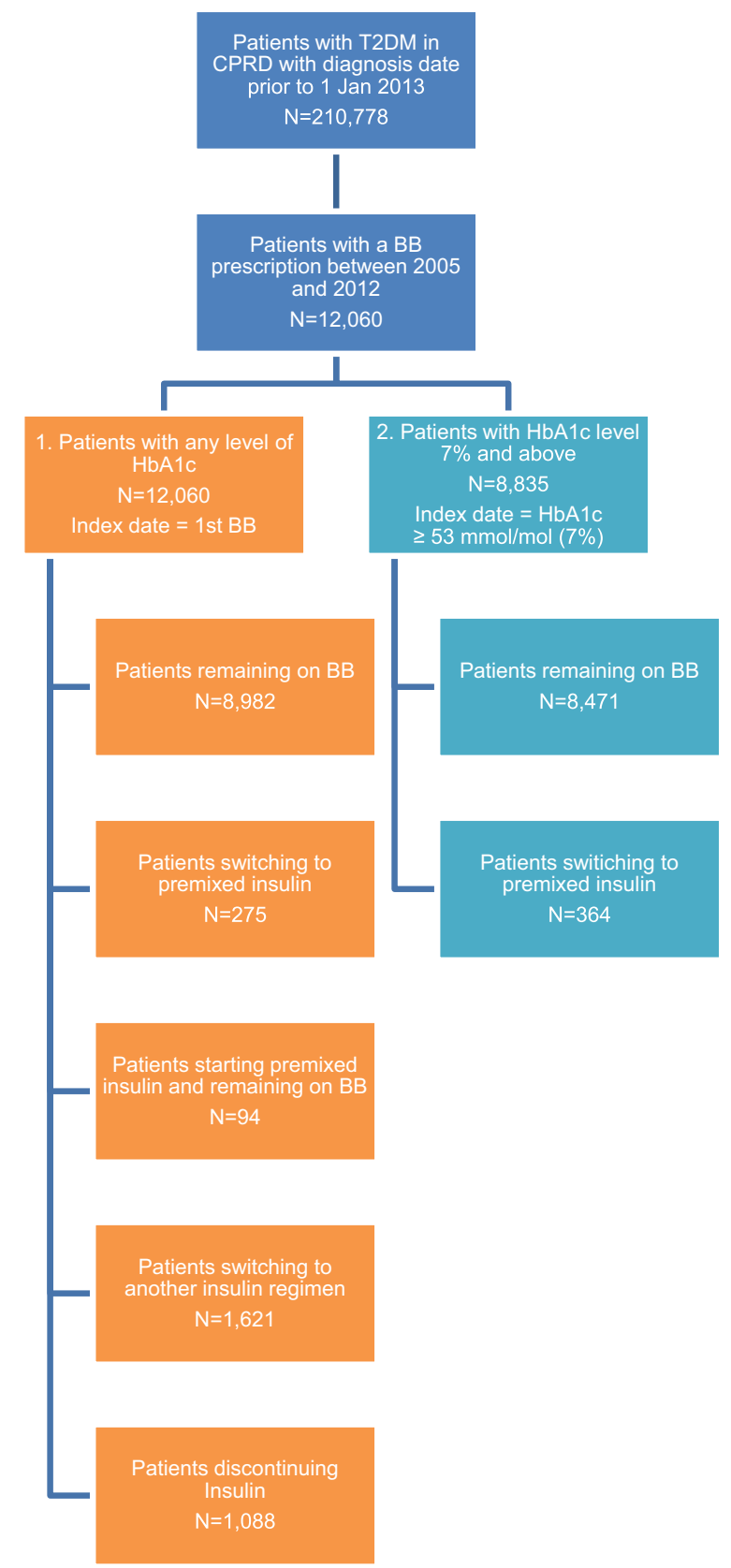

Abbreviations: BB, basal and bolus; $\mathrm{CPRD}$, Clinical Practice Research Datalink; HbA1c, haemoglobin A1c; T2DM, type 2 diabetes mellitus

Fig. 1 Sample selection flowchart for the comparative analyses included 8835 individuals with T2DM who did not achieve the glycaemic control target of a HbA1c level of $<53 \mathrm{mmol} / \mathrm{mol}$ (7.0\%) under their BB insulin regimen and who either remained on $\mathrm{BB}$ therapy (8471 patients, $95.9 \%)$ or who switched to a premixed insulin therapy (364 patients, $4.1 \%)$.

\section{Characteristics of Patients who Start BB Insulin}

Table 1 displays the demographic and clinical characteristics of patients with T2DM in the treatment sample. Patients treated with or initiating a BB regimen were on average 59 years old ( $\mathrm{SD} \pm 13$ years), were diagnosed with T2DM on average 12.4 (SD 7.5) years before starting $\mathrm{BB}$ insulin and had used other insulin products on average 3.6 (SD 3.5) years before starting their BB regimen. A substantial proportion of patients had diabetic retinopathy (35.0\%), coronary artery disease (24.0\%), peripheral vascular disease $(13.0 \%)$ or a combination of coronary artery disease, myocardial infarction or congestive heart failure $(26.0 \%)$ during the 12 months prior to starting $\mathrm{BB}$ insulin. More than half of the patients (59.0\%) were prescribed an $\mathrm{OAD}$, and a majority of these were using lipid-lowering (76.0\%), antihypertensive (66.0\%) and/or antithrombotic (55.0\%) medications. The average HbA1c level was $76 \mathrm{mmol} / \mathrm{mol}$ (9.1\%) (SD 1.7\%), with $73.0 \%$ having an HbA1c measure of $>64 \mathrm{mmol} / \mathrm{mol}(8.0 \%)$, and the average BMI was 31.2 (SD 6.6) $\mathrm{kg} / \mathrm{m}^{2}(84.0 \%$ were overweight or obese). Total cholesterol level was recorded in $47.5 \%$ of patients, with a mean measurement of of 3.6 (SD 1.0) $\mathrm{mmol} / \mathrm{L}$ (6.4\% had borderline-high or high cholesterol), and the mean systolic/diastolic blood pressure level for those with data recorded $(96.0 \%$ of 


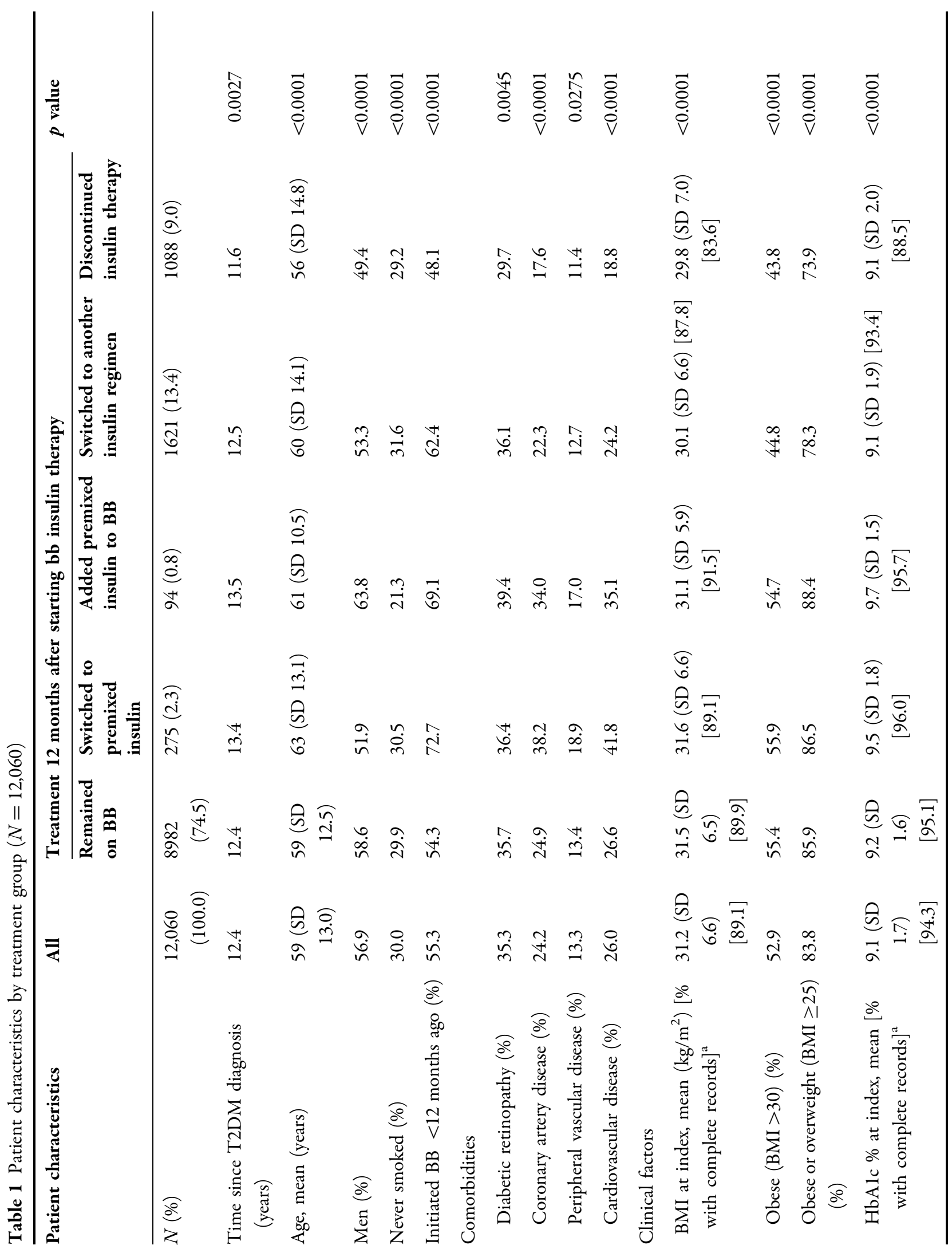




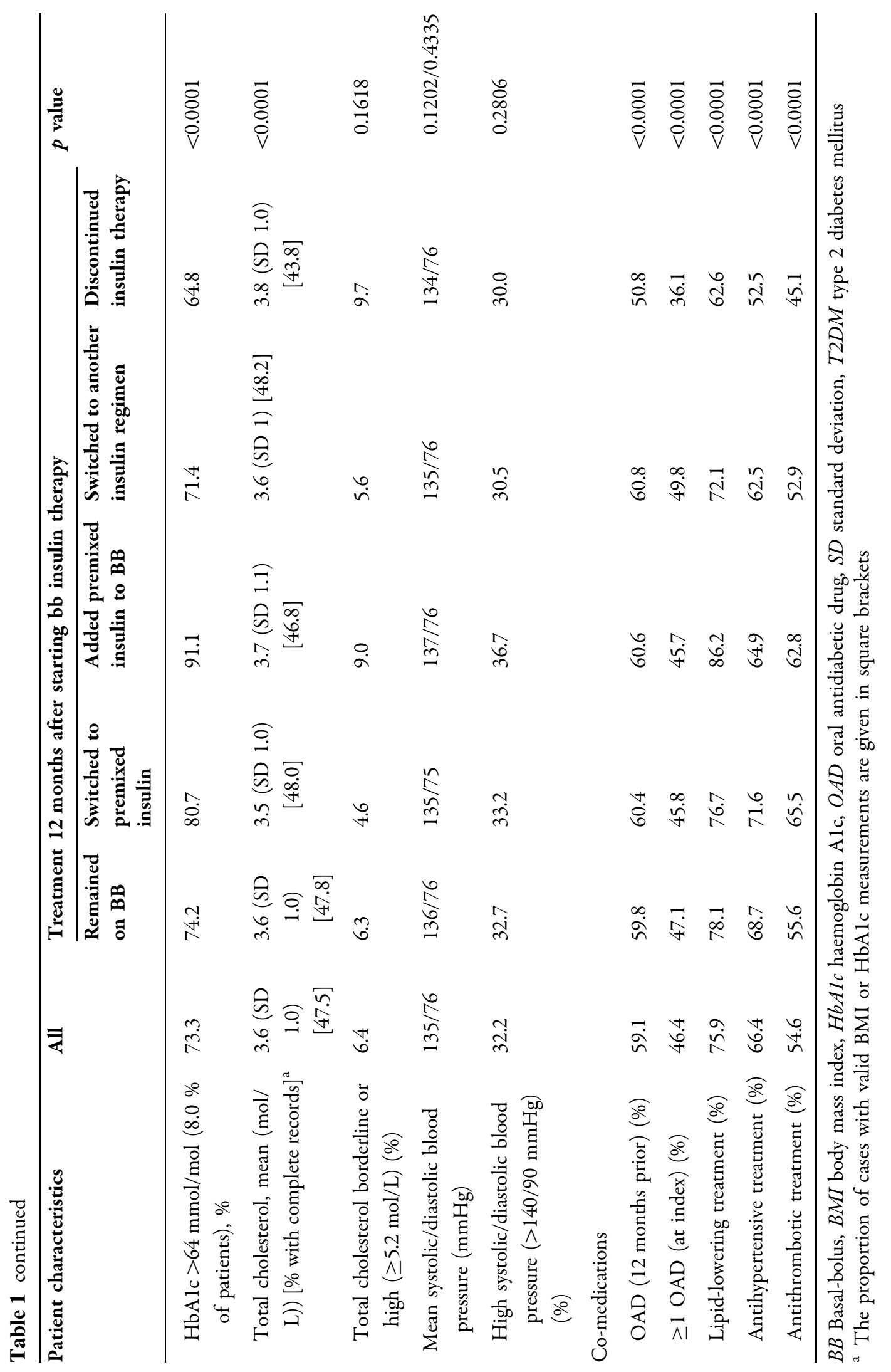


patients) was $135 / 76$ (SD 17/4) $\mathrm{mmHg}$, with $32.0 \%$ having a systolic/diastolic blood pressure level of $>140 / 90 \mathrm{mmHg}$.

\section{Treatment Patterns}

Table 1 also displays the characteristics of the sample by insulin-use patterns. Three-quarters of those who started BB therapy remained on it during the 12 months of follow-up, $13.0 \%$ switched to a primarily basal or bolus regimen and $9.0 \%$ discontinued insulin therapy. The remainder either switched to or supplemented their BB regimen with premixed insulin. Most patients who switched to premixed insulin or who added it to their BB treatment had initiated the $\mathrm{BB}$ regimen less than 12 months previously (72.7 and 69.1\%, respectively).

Patients discontinuing insulin therapy during the follow-up year were younger [56 (SD 14.8) years; $p<0.01]$ and diagnosed more recently [11.6 (SD 7.8) years] than other patients, while those switched to premixed insulin were older [63 (SD 13.1) years] and were diagnosed less recently [13.4 (SD 8.5) years; $p<0.01]$. Patients who discontinued insulin therapy were least likely to have any of the comorbidities included compared to other treatment groups, while patients who switched to premixed insulin were more likely to have had any of these. HbA1c levels were significantly different across groups $(p<0.01)$, with patients who added premixed insulin to their $\mathrm{BB}$ regimen $(91.1 \%)$ or switched to that regimen $(80.7 \%)$ most likely to have a HbA1c of $>64 \mathrm{mmol} / \mathrm{mol}$ ( $8.0 \%$ of patients). BMI was similar across the groups, but patients who added insulin to their BB regimen or who discontinued insulin were most likely to be overweight or obese (88.4 and 73.9\%, respectively, $p<0.01$ ).

\section{Comparative Analysis}

Table 2 displays baseline characteristics of the comparison analysis groups, and Figs. 2, 3, 4, 5 and 6 display the levels in clinical factors over time for patients with uncontrolled HbA1c [>53 $\mathrm{mmol} / \mathrm{mol} \quad(7.0 \%$ of patients)] who

Table 2 Patient characteristics by treatment group, comparative analysis $(N=8835)$

\begin{tabular}{|c|c|c|c|}
\hline Patient characteristics & $\begin{array}{l}\text { Switched to premixed } \\
\text { insulin }\end{array}$ & $\begin{array}{l}\text { Remained on } \\
\text { BB }\end{array}$ & $p$ value \\
\hline$N(\%)$ & $364(4.1)$ & $8471(95.9)$ & \\
\hline Duration of T2DM diagnosis, mean (years) & 13.0 SD (7.7) & $12.3(\mathrm{SD} 7.4)$ & $<0.1189$ \\
\hline Age, mean (years) & $61(\mathrm{SD} 12.9)$ & $58(\mathrm{SD} 12.4)$ & $<0.0001$ \\
\hline Men $(\%)$ & 46.2 & 58.4 & $<0.0001$ \\
\hline $\mathrm{HbAlc} 90$ days prior to index date, mean [\% with data recorded] & $9.8(\mathrm{SD} 1.7)[68.7]$ & $\begin{array}{l}8.8(\mathrm{SD} 1.4) \\
\quad[99.8]\end{array}$ & $<0.0001$ \\
\hline $\begin{array}{l}\text { Patients with } \mathrm{HbAlc}<64 \mathrm{mmol} / \mathrm{mol}(8.0 \%) 90 \text { days prior to } \\
\text { index date (\%) }\end{array}$ & 13.6 & 35.2 & $<0.0001$ \\
\hline $\begin{array}{l}\mathrm{BMI} \geq 30 \mathrm{~kg} / \mathrm{m}^{2} 90 \text { days prior to index date (\%) [\% with data } \\
\text { recorded] }\end{array}$ & $63.7[90.6]$ & $58.1[88.3]$ & 0.1215 \\
\hline
\end{tabular}

The proportion of patients with a valid BMI or HbAlc measurement at baseline are displayed in square brackets 


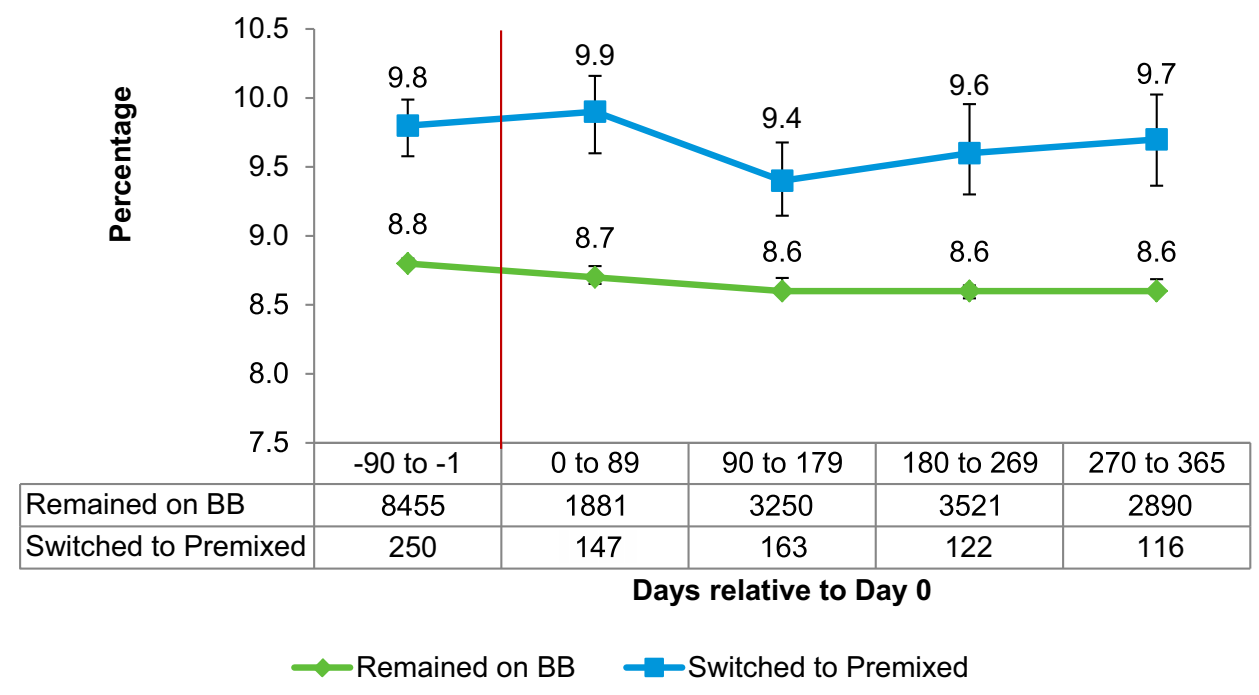

Abbreviations: BB; basal-bolus; HbA1c, haemoglobin A1c

Fig. 2 Haemoglobin Alc $(H b A 1 c)$ levels over time, by treatment group

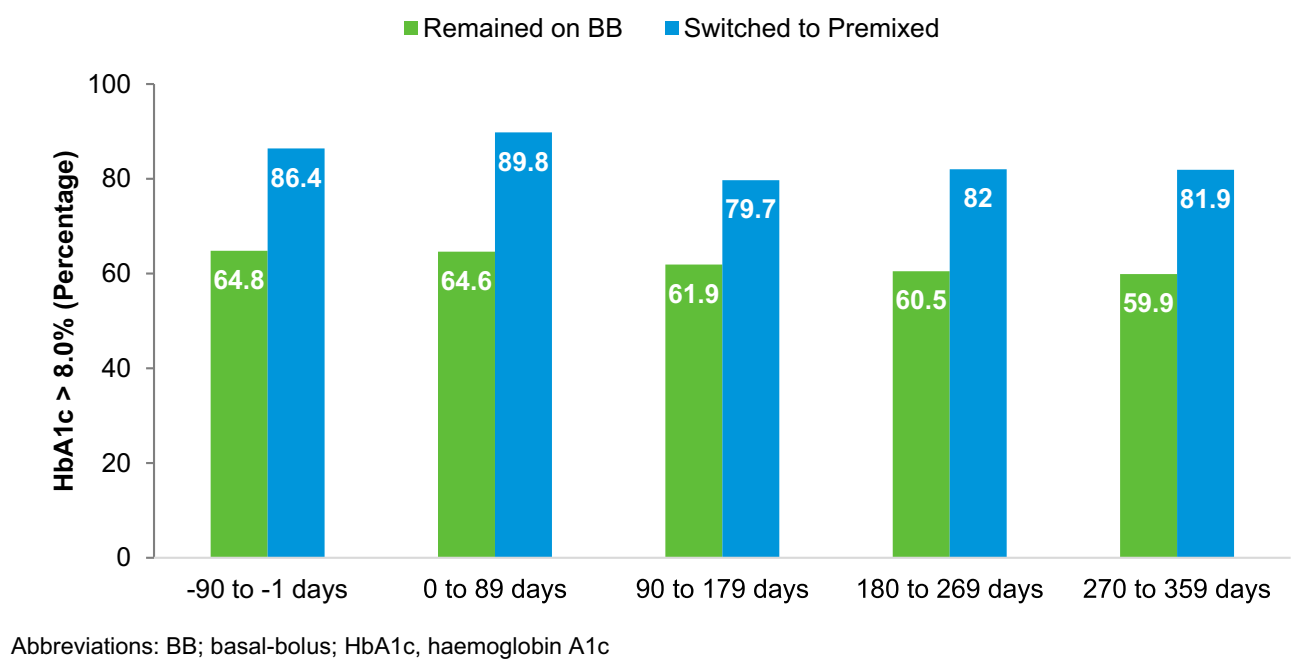

Fig. 3 Proportion of patients in the comparative sample with a $\mathrm{HbAlc}$ level of $>64 \mathrm{mmol} / \mathrm{mol}$ ( $8 \%$ of patients)

remained on their BB insulin regimen or who switched to premixed insulin. Patients remaining on $\mathrm{BB}$ insulin had on average lower baseline HbA1c levels than those who switched to premixed insulin: 73 versus $85 \mathrm{mmol} / \mathrm{mol}$ $[8.8 \%$ (SD $1.4 \%$ ) vs. $9.8 \%$ (SD $1.7 \%$ ), respectively]. Among patients with a HbA1c level of $<64 \mathrm{mmol} / \mathrm{mol}(8.0 \%), 35.2 \%$ remained on BB compared to $13.6 \%$ who switched to premixed insulin. On average, the BMI was lower for patients remaining on $\mathrm{BB}$ than for those who switched to premixed insulin [32.0 (SD 6.6) vs. 33.1 (SD 6.4) $\mathrm{kg} / \mathrm{m}^{2}$, respectively], with $88.3 \%$ of patients remaining on $\mathrm{BB}$ versus $90.6 \%$ of patients switching to premixed insulin with a BMI of $\geq 25.0 \mathrm{~kg} / \mathrm{m}^{2}$. Median total cholesterol levels were similar in the groups of patients who remained on their BB insulin 


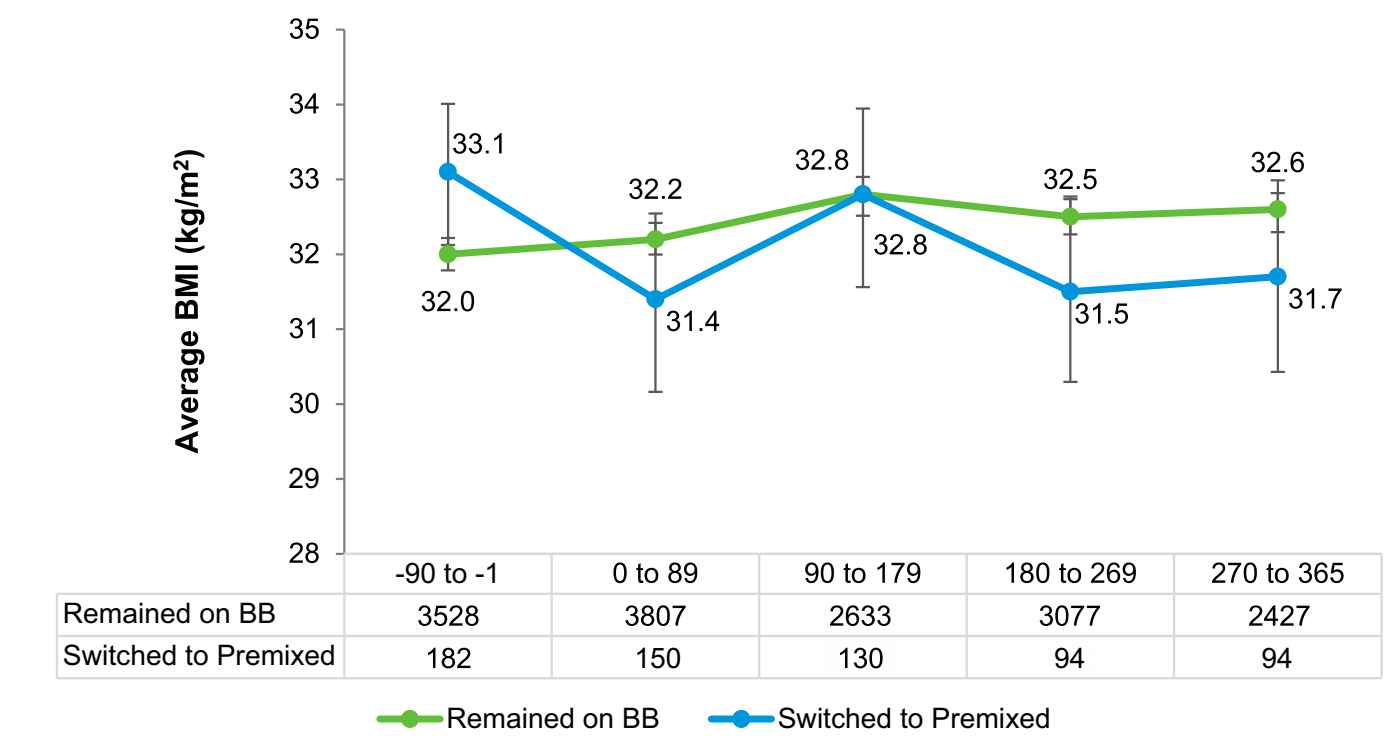

Abbreviations: BB; basal-bolus; BMI, body mass index

Fig. 4 Body mass index $(B M I)$ levels over time, by treatment group

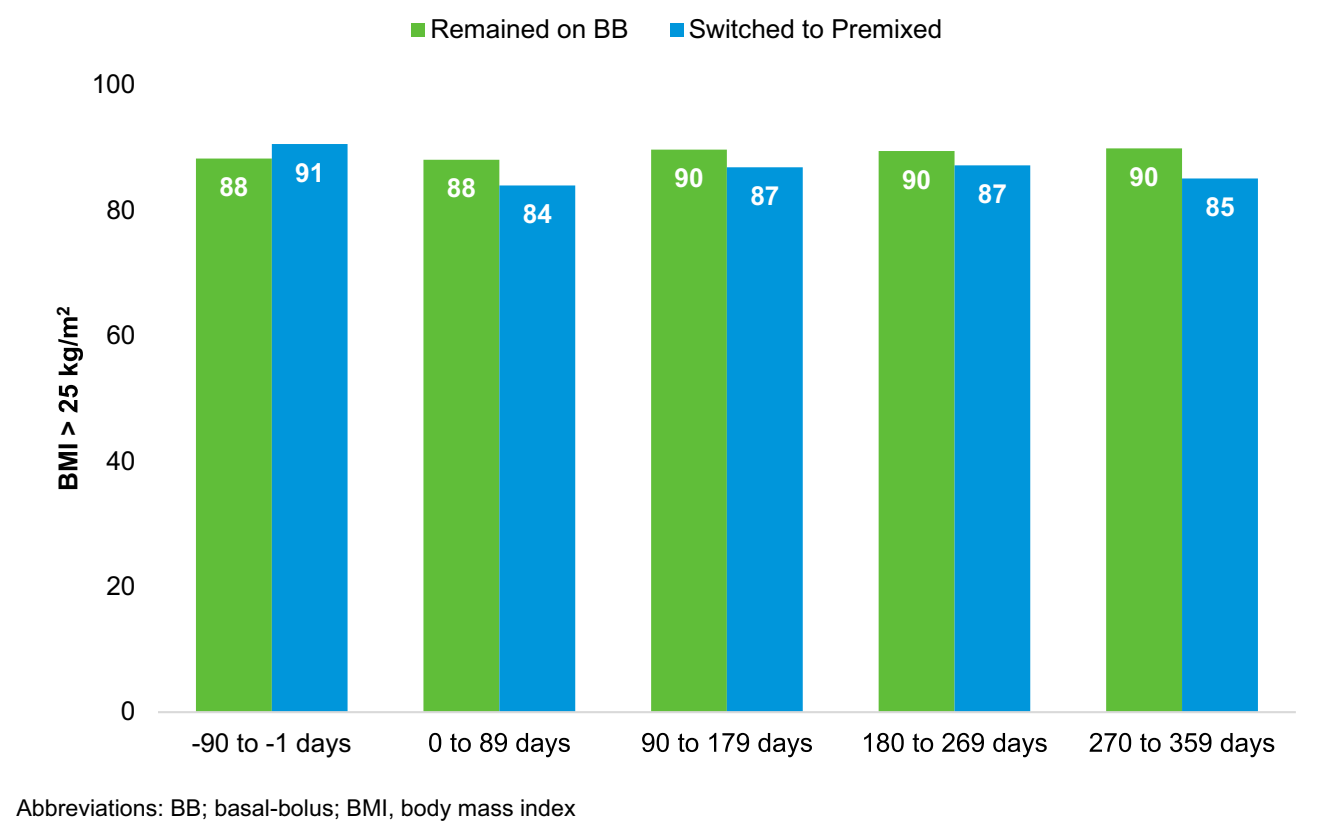

Fig. 5 Proportion of patients in the comparative sample with a BMI of $>25 \mathrm{~kg} / \mathrm{m}^{2}$, by treatment group

regimen or who switched to premixed insulin [3.5 (IQR 2.9-4.2) vs. 3.2 (IQR 2.6-4.1) $\mathrm{mmol} / \mathrm{L}$, respectively].

Although average HbA1c levels and the prevalence of a HbA1c level of $>64 \mathrm{mmol} / \mathrm{mol}$
(8.0\%) were consistently higher for patients who switched to premixed insulin than for those remaining on their $\mathrm{BB}$ insulin regimens ( $p<0.01$ for all time intervals), they remained relatively unchanged over time (Figs. 2, 3). 


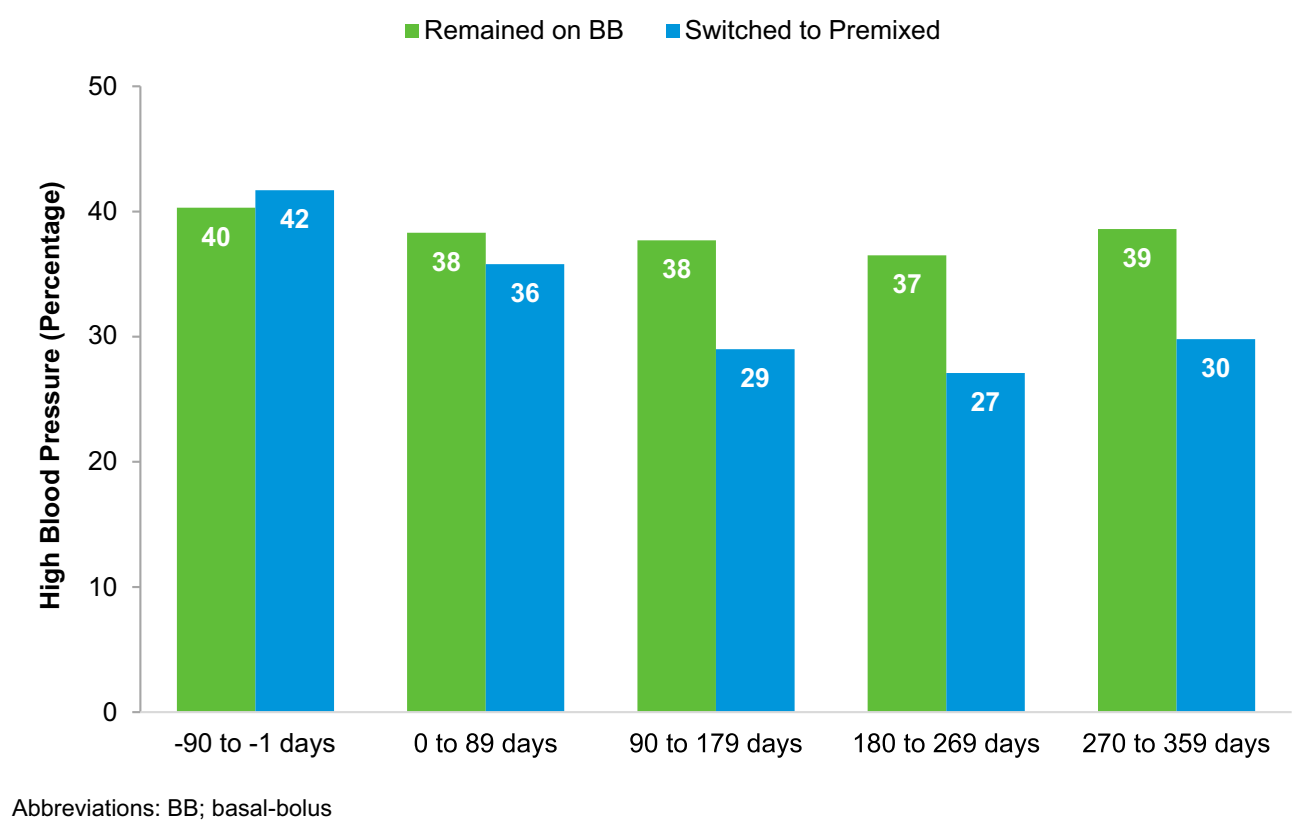

Fig. 6 Proportion of patients in the comparative sample with diastolic blood pressure of $>90 \mathrm{mmHg}$ and a systolic blood pressure of $>140 \mathrm{mmHg}$, by treatment group

Patients who switched to premixed insulin tended to have higher BMI values and were more likely to be overweight at baseline than those who remained on their $\mathrm{BB}$ regimen $(p<0.05)$. The differences in BMI and the prevalence of overweight patients between the two groups varied greatly during other time periods (Figs. 4, 5). The proportion of patients with systolic/diastolic blood pressure records of $>140 / 90 \mathrm{mmHg}$ was similar between both groups but diverged over time so that by the end of follow-up, the proportion of patients with high blood pressure readings among patients who switched was almost 25.0\% lower than that of patients who remained on their BB regimen $(p=0.05$ during the last 90 -day interval) (Fig. 6).

\section{DISCUSSION}

This two-part analysis used a large UK primary care database to investigate clinical and demographic characteristics and treatment patterns for a sample of patients with T2DM who started a BB insulin regimen and for the subgroup of patients who did not achieve HbA1c targets on BB insulin in order to compare these characteristics between those who subsequently continued in this regimen and those who switched to premixed insulin. The proportion of patients treated with a $\mathrm{BB}$ regimen was consistent with previous studies that estimated $4.0 \%$ of patients in the UK initiating insulin therapy between 2004 and 2006 were treated with this regimen [19].

This study showed that in the UK, three-quarters of patients with T2DM who started BB insulin therapy remained on it in the next year. Fewer patients (9.0\%) discontinued insulin after 1 year and $3.1 \%$ of patients switched to premixed insulin or added premixed insulin to their BB regimen. Patients who later switched to premixed insulin or who added it to their $\mathrm{BB}$ regimen were on average 
older, had higher baseline HbA1c levels and were more likely to have comorbid conditions compared to those who remained in the BB regimen or were classified in the other treatment pattern groups.

At the start of the study, only $8.3 \%$ of the prevalent patients with $\mathrm{T} 2 \mathrm{D}$ receiving $\mathrm{BB}$ had an HbA1c concentration of less than the $53 \mathrm{mmol} / \mathrm{mol}(7.0 \%)$ target in the 12 months prior. The inability to achieve good glycaemic control in clinical practice with insulin therapy has previously been described; Nichols et al. $[20,21]$ found that only $30.0-40.0 \%$ of insulin-treated patients were well-controlled; further, Nichols et al. [20] showed that pre-insulin levels of $\mathrm{HbA1c}$ were the main factor in determining whether the target HbA1c level was reached after insulin initiation. The patients in this study remained at high HbA1c levels irrespective of whether they remained on their BB insulin regimen or switched to a premixed insulin. Even in randomised controlled clinical trials, less than half of the patients with T2DM treated with $\mathrm{BB}$ were able to achieve an HbA1c level of $<7.0 \%$ [22].

The second part of our study then focused on patients who did not achieve HbA1c levels of $<7.0$ with $\mathrm{BB}$ and either remained on that regimen or switched to premixed insulin. All clinical factors investigated indicated that the group of patients who switched to premixed insulin were less well-controlled. The HbA1c and BMI measurements at 12 months of follow-up showed that the HbA1c concentration and BMI remained fairly constant for both groups. Despite the difference between the groups at baseline, we found little evidence that the type of insulin therapy was associated with any meaningful changes in key clinical outcomes over time. This result suggests that the simplified regimen afforded by premixed insulin allowed patients who switched therapeutic regimen to maintain similar HbA1c levels to that achieved with a BB regimen prior to the switch.

While BB therapy is regarded as the gold standard for insulin therapy, its benefit may only appear if the patient understands the technicalities of such a complex regimen, including the need for frequent blood glucose monitoring and insulin dose adjustments. Donnelly et al. [11] showed that adherence to insulin was a significant predictor of HbA1c. Similarly, the ADA/EASD approach to starting and adjusting insulin shows that BB therapy might not be the best 'end of line' therapy for many, and premixed insulin may be an acceptable alternative in achieving glycaemic control [4].

A recent meta-analysis by Wang et al. [14] found that among insulin-naïve patients with T2DM, premixed insulin was equally as effective as BB insulin in reducing HbA1c. In a UK-wide evaluation in the secondary care setting, the clinical use of biphasic insulin lispro 50/50 showed the greatest $\mathrm{HbA1c}$ reduction in those patients who were previously treated with a $\mathrm{BB}$ regimen [13]. Reasons for remaining on BB therapy whilst having high HbA1c levels were not investigated in this study. The ADA/EASD guidelines recommend a patient-centred approach in the choice of pharmacological agents, including considerations on efficacy, cost, potential side effects, weight, comorbidities, hypoglycaemia risk and patient preferences [4]. Similarly, in the UK, the NICE guidelines for the management of T2DM in adults [3] recommend that an individualised approach to diabetes care is followed, which includes personal preferences, comorbidities and the risk of polypharmacy.

As GPs are the main managers of T2DM in the UK, the strengths of this study include the 
use of a large primary care database representative of the UK population with a range of available outcome measures [23]. To our knowledge this study is the first to describe outcomes of patients switching from BB to a premixed insulin regimen in the primary care setting. The findings are consistent with those of other studies looking at glycaemic control in that they show that patients with high HbA1c levels find it difficult to reach target levels irrespective of the treatment type [20-22].

Still the use of CPRD data carried some limitations in terms of data availability. The characteristics of patients who intensified their treatment regimen with higher insulin doses or additional bolus injections could not be investigated in this study given the unavailability of this information in the database. The electronic medical records used did not contain sufficient data on factors influencing medication choice, such as lifestyle, patient preference and extent of information given to the patient on available medication. While information on prescriptions is available in the database, there are no records of adherence to treatment which could affect outcomes.

A methodological limitation of this comparative analysis, which sought to understand the effect of switching to premixed insulin, was that the subgroups of patients who remained on their $\mathrm{BB}$ regimens and those who switched to premixed insulin did not have comparable baseline demographic and clinical characteristics. The differences observed in outcomes could be due to factors other than insulin regimen, and the relatively small sample of patients with sufficient follow-up data and absence of information on other behavioural predictive factors precluded the use of multivariate regression or matching methods to address potential confounding.

\section{CONCLUSION}

The study findings indicate that for patients with $\mathrm{T} 2 \mathrm{D}$ receiving insulin, clinicians can consider the option of premixed insulin for patients not achieving the target glycaemic control and for those who will benefit from a less complex regimen. Further research to better understand the options to improve glycaemic control, including therapy change to a less complex regimen, for patients not achieving target HbA1c levels with BB therapy would benefit an informed clinical decision.

\section{ACKNOWLEDGEMENTS}

Sponsorship and article processing charges for this study were funded by Eli Lilly and Company. M. Raluy-Callado, C. Chalkiadaki and S. MacLachlan were involved in the preparation of the data and interpretation of results. Together with D. Neasham, K. van Brunt, B. Curtis, T. Ivanyi and E. Balogh, they were involved in drafting and revising the article. All authors approved the final version to be published and are accountable for all aspects of the work. The authors want to thank Michael Ganz, Research Scientist at Evidera, for his review and editing of this manuscript, and Robert Donaldson, Data Analyst at Evidera, for his role in preparing the data and producing the majority of the results included in this article. All named authors meet the International Committee of Medical Journal Editors (ICMJE) criteria for authorship for this manuscript, take responsibility for the integrity of the work as a whole, and have given final approval for the version to be published. Prior presentationAbstract selected for publication only in the American Diabetes Association 75th Scientific 
Sessions Abstract Book June 2015-Reference 2762-PO.

Data Availability. The datasets analyzed during the current study are not publicly available as our licence agreement with CPRD states that we shall not permit any third party to access, study, analyse, refer to or otherwise use the dataset or parts thereof.

Disclosures. M. Raluy-Callado was a consultant paid by Eli Lilly to conduct this study. C. Chalkiadaki was a consultant paid by Eli Lilly to conduct this study. D. Neasham was a consultant paid by Eli Lilly to conduct this study. S. MacLachlan was a consultant paid by Eli Lilly to conduct this study. K. van Brunt is an employee of Eli Lilly. B. Curtis is an employee of Eli Lilly. T. Ivanyi is an employee of Eli Lilly. E. Balogh is an employee of Eli Lilly.

Compliance with Ethics Guidelines. This study was approved by the Independent Scientific Advisory Committee (ISAC) of the Medicines and Healthcare products Regulatory Agency (MHRA) Database Research (Protocol Number 13_220). This article does not contain any new studies with human or animal subjects performed by any of the authors.

Open Access. This article is distributed under the terms of the Creative Commons Attribution-NonCommercial 4.0 International License (http://creativecommons.org/licenses/ by-nc/4.0/), which permits any noncommercial use, distribution, and reproduction in any medium, provided you give appropriate credit to the original author(s) and the source, provide a link to the Creative Commons license, and indicate if changes were made.

\section{REFERENCES}

1. Diabetes UK. Diabetes in the UK: Key facts and statistics on diabetes. Revised: November 2015. http://www.diabetes.org.uk/. Accessed 15 Jan 2016.

2. Holman N, Young B, Gadsby R. Current prevalence of Type 1 and Type 2 diabetes in adults and children in the UK. Diabet Med. 2015;32(9):1119-20.

3. National Institute for Health and Care Excellence (NICE). Type 2 diabetes in adults: management. NICE guidelines NG28. 2015. http://www.nice.org.uk/guidance/ng28. Accessed 15 Jan 2016.

4. Inzucchi SE, Bergenstal RM, Buse JB, Diamant M, Ferrannini E, Nauck $M$, et al. Management of hyperglycemia in type 2 diabetes, 2015: a patient-centered approach: update to a position statement of the American Diabetes Association and the European Association for the Study of Diabetes. Diabetes Care. 2015;38(1):140-9.

5. Khunti K, Nikolajsen A, Thorsted BL, Andersen M, Davies MJ, Paul SK, et al. Clinical inertia in intensifying therapy among people with type 2 diabetes treated with basal insulin. Diabetes Obes Metab. 2016;18(4):401-9.

6. Holman RR, Thorne KI, Farmer AJ, Davies MJ, Keenan JF, Paul S, et al. Addition of biphasic, prandial, or basal insulin to oral therapy in type 2 diabetes. N Engl J Med. 2007;357(17):1716-30.

7. Holt RI. Insulin initiation in Type 2 diabetes: the implications of the 4-T study. Diabet Med. 2010;27(1):1-3.

8. Gallagher N, Cardwell C, Hughes C, O'Reilly D. Increase in the pharmacological management of Type 2 diabetes with pay-for-performance in primary care in the UK. Diabet Med. 2015;32(1):62-8.

9. Khunti K, Wolden ML, Thorsted BL, Andersen M, Davies MJ. Clinical inertia in people with type 2 diabetes: a retrospective cohort study of more than 80,000 people. Diabetes Care. 2013;36(11):3411-7.

10. Kunt T, Snoek FJ. Barriers to insulin initiation and intensification and how to overcome them. Int J Clin Pract Suppl. 2009;63[Suppl 164]:6-10.

11. Donnelly LA, Morris AD, Evans JM, DARTS/ MEMO collaboration. Adherence to insulin and its association with glycaemic control in patients with type 2 diabetes. QJM. 2007;100(6):345-50. 
12. Nathan DM, Buse JB, Davidson MB, Ferrannini E, Holman RR, Sherwin R, et al. Medical management of hyperglycemia in type 2 diabetes: a consensus algorithm for the initiation and adjustment of therapy: a consensus statement of the American Diabetes Association and the European Association for the Study of Diabetes. Diabetes Care. 2009;32(1):193-203.

13. Mungreiphy NK, Mamza J, Lakhdar AF, Bannister M, Elliott J, Idris I. Clinical use and efficacy of biphasic insulin lispro 50/50 in people with insulin treated diabetes-a nationwide evaluation of clinical practice. Curr Med Res Opin. 2015;31(3):493-501.

14. Wang C, Mamza J, Idris I. Biphasic vs basal bolus insulin regimen in Type 2 diabetes: a systematic review and meta-analysis of randomized controlled trials. Diabet Med. 2015;32(5):585-94.

15. Fitzsimons B, Wilton L, Lamont T, McCulloch L, Boyce J. The Audit Commission review of diabetes services in England and Wales, 1998-2001. Diabet Med. 2002;19(Suppl 4):73-8.

16. Department of Health. National service framework for diabetes: delivery strategy. Department of Health, London; 2003.

17. Khan NF, Harrison SE, Rose PW. Validity of diagnostic coding within the General Practice Research Database: a systematic review. Br J Gen Pract. 2010;60(572):e128-36.
18. Herrett E, Thomas SL, Schoonen WM, Smeeth L, Hall AJ. Validation and validity of diagnoses in the General Practice Research Database: a systematic review. Br J Clin Pharmacol. 2010;69(1):4-14.

19. Blak BT, Smith HT, Hards M, Curtis BH, Ivanyi T. Optimization of insulin therapy in patients with type 2 diabetes mellitus: beyond basal insulin. Diabet Med. 2012;29(7):e13-20.

20. Nichols GA, Kimes TM, Harp JB, Kou TD, Brodovicz KG. Glycemic response and attainment of A1C goals following newly initiated insulin therapy for type 2 diabetes. Diabetes Care. 2012;35(3):495-7.

21. Nichols GA, Gandra SR, Chiou CF, Anthony MS, Alexander-Bridges M, Brown JB. Successes and challenges of insulin therapy for type 2 diabetes in a managed-care setting. Curr Med Res Opin. 2010;26(1):9-15.

22. Lind M, Hirsch IB, Tuomilehto J, Dahlqvist S, Ahren $B$, Torffvit $\mathrm{O}$, et al. Liraglutide in people treated for type 2 diabetes with multiple daily insulin injections: randomised clinical trial (MDI Liraglutide trial). BMJ. 2015;351:h5364.

23. Garcia Rodriguez LA, Perez Gutthann S. Use of the UK General Practice Research Database for pharmacoepidemiology. Br J Clin Pharmacol. 1998;45(5):419-25. 